Homology, Homotopy and Applications, vol.4(2), 2002, pp.427-437

\title{
RESOLUTIONS AND LATTICES
}

\author{
IRENA PEEVA
}

(communicated by Clas Löfwall)

\begin{abstract}
We discuss how lattices and posets can be used as tools to study minimal free resolutions of monomial or toric ideals.
\end{abstract}

To Jan-Erik Roos on his sixty-fifth birthday

\section{Introduction}

In this paper we discuss properties, related to certain lattices and posets, of monomial and toric resolutions.

A lattice is called a finite geometric lattice if it is finite, semimodular, and atomic. The significance of such lattices comes from the fact that a lattice $L$ is a finite geometric lattice if and only if $L$ is the intersection lattice of a central essential hyperplane arrangement, if and only if $L$ is the lattice of flats of a simple matroid. We call a monomial ideal $M$ geometric if its lcm-lattice is geometric. In Section 2, we construct the minimal free resolution $\mathbf{F}_{M}$ of $S / M$ as a cellular (simplicial in this case) algebraic complex; this provides a large class of ideals whose minimal free resolutions are simplicial but bigger than the Scarf-complex resolution. We also construct $\mathbf{F}_{M}$ as a quotient of Taylor's resolution. A nice application is Theorem 2.10, which shows that the Poincaré polynomial of the intersection lattice of a central hyperplane arrangement is equal to the Poincaré series of the minimal free resolution of a certain monomial ideal. It leads to a dictionary between some questions/invariants of simple matroids and questions/invariants of minimal free resolutions of geometric ideals. Also, our approach leads to a new (algebraic) proof of Corollary 2.11, which is an important fact in matroid theory and was proved in different ways by Björner, Gelfand-Zelevinsky, Jambu-Terao.

In Section 3 we consider Betti numbers of toric ideals. M. Hochster and R. Stanley have found and proved that such Betti numbers can be computed using simplicial complexes. That formula for the Betti numbers was first published in the first edition of [St] (cf. [St, Theorem 7.9]). For a proof of the formula and applications, see cf. $[\mathrm{BH}]$. We construct a different type of simplicial complexes in Construction 3.1, and we prove in Theorem 3.4(b) that they provide the Betti numbers as well; these simplicial complexes were first introduced in discussions between D. Bayer, B. Sturmfels, and the author. Recently, the computation using lattices of

I thank the referee for suggesting the short proof of Theorem 2.2.

Received February 16, 2001, revised January 7, 2002; published on July 12, 2002.

2000 Mathematics Subject Classification: 13D02.

Key words and phrases: Syzygies.

(c) 2002, Irena Peeva. Permission to copy for private use granted. 
the Betti numbers of a monomial ideal was developed in [GPW, Theorem 2.1]. We introduce the computation of the Betti numbers of a toric ideal by posets in Construction 3.3 and Theorem 3.4(b). We also discuss the homotopy equivalence between the simplicial complexes and posets that can be used to obtain the Betti numbers. The proof of Theorem 3.4 uses methods from Topological Combinatorics [Bj]. The homotopy equivalence of the complexes $X(M)$ and $\Gamma(M)$ in Theorem 3.4 can be proved alternatively using Theorem 3.5 on monomial ideals and the methods in [BS]; our proof of this homotopy equivalence does not rely on any results on monomial ideals and is much simpler than the methods developed in [BS].

\section{Geometric monomial ideals}

Let $S=k\left[x_{1}, \ldots, x_{n}\right]$ be the polynomial ring over a field $k$. In this section $M$ stands for a geometric monomial ideal minimally generated by monomials $m_{1}, \ldots, m_{r}$. We will construct the minimal free resolution of $S / M$.

According to [GPW] the lcm-lattice $L$ of $M$ is the lattice with elements labeled by the least common multiples of $m_{1}, \ldots, m_{r}$ ordered by divisibility; in particular the atoms in $L$ are $m_{1}, \ldots, m_{r}$, the maximal element is $\operatorname{lcm}\left(m_{1}, \ldots, m_{r}\right)$, and the minimal element is 1 regarded as the lcm of the empty set.

If $l_{1}, \ldots, l_{p}$ are elements, then we denote by $l_{1} \vee \cdots \vee l_{p}=\operatorname{lcm}\left(l_{1}, \ldots, l_{p}\right)$ the join of these elements. The degree of a set $J \subset[r]$ is $m_{J}=\operatorname{lcm}\left(m_{j} \mid j \in J\right)$. We say that $J$ is independent if $m_{J} \neq m_{J \backslash a}$ for each $a \in J$. A set $J$ is dependent if it is not independent. The minimal dependent sets are called circuits. If $C$ is a circuit and $c$ is the element in $C$ with the smallest index, then $C \backslash c$ is called a broken circuit. A set $J$ is called a nbc-set if it contains no broken circuit. The nbc-sets form a simplicial complex bc $(M)$ called the broken circuit complex. Note that in the construction of $\mathrm{bc}(M)$ we used the order of the minimal monomial generators $m_{1}, \ldots, m_{r}$; changing the order of $m_{1}, \ldots, m_{r}$ might change the broken circuit complex.

Construction 2.1. We build a cellular (simplicial in this case) resolution of $S / M$ applying Construction 2.1 from [BPS] to bc $(M)$. Let $E$ be the exterior algebra over $k$ on basis elements $e_{1}, \ldots, e_{r}$. We consider Taylor's (possibly non-minimal) resolution $\mathbf{E}$, which is the module $S \otimes E$ equipped with the differential

$$
d\left(e_{j_{1}} \wedge \cdots \wedge e_{j_{s}}\right)=\sum_{1 \leqslant i \leqslant s}(-1)^{i+1} \cdot \frac{m_{J}}{m_{J \backslash j_{i}}} \cdot e_{j_{1}} \wedge \cdots \wedge \widehat{e}_{j_{i}} \wedge \cdots \wedge e_{j_{s}},
$$

where $J=\left\{j_{1}, \ldots, j_{s}\right\}$ and $\widehat{e}_{j_{i}}$ means that $e_{j_{i}}$ is omitted in the product. Taylor's resolution is multigraded with $\operatorname{deg}\left(e_{j_{1}} \wedge \cdots \wedge e_{j_{s}}\right)=\operatorname{lcm}\left(m_{j_{1}}, \ldots, m_{j_{s}}\right)$ and homologically graded by $\operatorname{deg}\left(e_{j_{1}} \wedge \cdots \wedge e_{j_{s}}\right)=s$. Set $\mathbf{F}_{M}$ to be the subcomplex of $\mathbf{E}$ generated as an $S$-module by the elements $\left\{e_{j_{1}} \wedge \cdots \wedge e_{j_{s}} \mid\left\{j_{1}, \ldots, j_{s}\right\}\right.$ is an nbc-set $\}$.

Theorem 2.1. If $M$ is geometric, then $\mathbf{F}_{M}$ is the minimal free resolution of $S / M$.

Proof. The complex $\mathbf{F}_{M}$ constructed in 2.1 coincides with Lyubeznik's resolution [Ly]. Thus, $\mathbf{F}_{M}$ is exact. Since the lcm-lattice is geometric, we have that each nbcset is an independent set. Hence, $d\left(\mathbf{F}_{M}\right) \subset\left(x_{1}, \ldots, x_{n}\right) \mathbf{F}_{M}$, which means that the resolution is minimal. 
Next we take a completely different approach and build the minimal free resolution of $S / M$ as a quotient of Taylor's resolution.

Construction 2.2. We will construct the Orlik-Solomon complex of $M$ as a quotient of Taylor's resolution by a free submodule; it will be a complex of free modules homologically graded and multigraded.

We consider Taylor's (possibly non-minimal) resolution $\mathbf{E}$ described in Construction 2.1. If $J=\left\{j_{1}, \ldots, j_{s}\right\} \subseteq[n]$ and $j_{1}<\cdots<j_{s}$, then we denote by $e_{J}$ the element $e_{j_{1}} \wedge \cdots \wedge e_{j_{s}}$.

Denote by $L$ the lcm-lattice of $M$. We call $e_{j_{1}} \wedge \cdots \wedge e_{j_{s}}$ a circuit element, dependent element, or independent element if $\left\{j_{1}, \ldots j_{s}\right\}$ is a circuit, dependent set, or independent set, respectively. Set $\mathbf{D}$ to be the $S$-submodule of $\mathbf{E}$ generated by all dependent elements. The independent sets form a simplicial complex, hence $d$ (independent element) is an $S$-combination of independent elements.

If $C$ is a circuit, then

$$
d\left(e_{j_{1}} \wedge \cdots \wedge e_{j_{s}}\right)=\sum_{1 \leqslant i \leqslant s}(-1)^{i+1} e_{j_{1}} \wedge \cdots \wedge \widehat{e}_{j_{i}} \wedge \cdots \wedge e_{j_{s}} .
$$

Set $\mathbf{C}$ to be the S-submodule of $\mathbf{E}$ generated by all d(circuit element). Clearly, $d(\mathbf{C})=0$.

Note that $\mathbf{D} \oplus \mathbf{C}$ is an ideal in $\mathbf{E}$. Furthermore, we have the inclusion $d(\mathbf{D} \oplus \mathbf{C}) \subseteq$ $\mathbf{D} \oplus \mathbf{C}$ because $d(\mathbf{C})=0$ and if $e_{J} \in \mathbf{D}$ then

$$
d\left(e_{J}\right) \in \begin{cases}\mathbf{C} & \text { if Jisacircuit, } \\ \mathbf{D} & \text { if Jisdependent, butnotacircuit. }\end{cases}
$$

We call the complex $\mathbf{F}_{\text {os }(M)}=\mathbf{E} /(\mathbf{D} \oplus \mathbf{C})$ the Orlik-Solomon complex of $M$. It is a free $S$-module. For every circuit $C$ we have that $d\left(e_{C}\right)$ is homogeneous with respect to the multigrading and the homological grading; so $\mathbf{F}_{o s(M)}$ is graded by homological degree and multigraded.

Theorem 2.2. If $M$ is geometric, then $\mathbf{F}_{o s(M)}$ is the minimal free resolution of $S / M$. This resolution is a differential graded algebra.

Proof. First, we will prove that $\mathbf{F}_{o s(M)}$ is exact. For an element $\alpha \in \mathbf{E}$ we denote by $\bar{\alpha}$ its image in $\mathbf{F}_{o s(M)}=\mathbf{E} /(\mathbf{D} \oplus \mathbf{C})$. Choose a homogeneous element $\alpha \in \mathbf{E}$ such that $d(\bar{\alpha})=0$, that is $d(\alpha) \in \mathbf{C}$. Therefore, there exist circuit elements $\beta_{1}, \ldots, \beta_{q}$ and coefficients $\mu_{1}, \ldots, \mu_{q}$ such that $d(\alpha)=d\left(\sum_{1 \leqslant i \leqslant q} \mu_{i} \beta_{i}\right)$. Since Taylor's resolution $\mathbf{E}$ is exact, it follows that there exists a $\gamma \in \mathbf{E}$ such that

$$
\alpha=\sum_{1 \leqslant i \leqslant q} \mu_{i} \beta_{i}+d(\gamma)
$$

Thus, $\bar{\alpha}=\overline{d(\gamma)}$. We have $\bar{\alpha}=d(\bar{\gamma})$ as desired. Hence $\mathbf{F}_{o s(M)}$ is a free resolution of $S / M$.

By Construction 2.4, we have $d\left(\mathbf{F}_{o s(M)}\right) \subseteq\left(x_{1}, \ldots, x_{n}\right) \mathbf{F}_{o s(M)}$. Therefore, the resolution $\mathbf{F}_{o s(M)}$ is minimal.

Taylor's resolution $\mathbf{E}$ is a differential graded algebra. Since, $\mathbf{D} \oplus \mathbf{C}$ is an ideal preserved under the differential, it follows that $\mathbf{F}_{o s(M)}$ is a differential graded algebra as well. 
A lattice is a finite geometric lattice if and only if it is the lattice of flats of a simple matroid, if and only if it is the intersection lattice of a central essential hyperplane arrangement. In the latter correspondence, the ground set of the simple matroid is identified with the atoms (the hyperplanes in the arrangement) in the intersection lattice of the arrangement. In this section $\mathcal{A}$ stands for a central essential hyperplane arrangement in $k^{q}$ with $r$ hyperplanes. Denote by $L$ the intersection lattice of $\mathcal{A}$.

Construction 2.3. Note that $L$ is atomic and coatomic. We will construct a monomial ideal $M$, such that $L$ is its lcm-lattice. Let the atoms of $L$ be labeled by $\overline{1}, \ldots, \bar{r}$. Label the coatoms of $L$ by $1^{\prime}, \ldots, n^{\prime}$. Label an atom $\bar{i}$ by the monomial

$$
m_{i}=\prod_{\bar{i} \vee j^{\prime}=\hat{1}} x_{j} .
$$

Let $M$ be the monomial ideal generated by the monomial labels of the atoms. If there exist two generators $m_{p}$ and $m_{q}$ such that $m_{p}$ divides $m_{q}$, then $\left\{j \mid \bar{q}<j^{\prime}\right\} \subset$ $\left\{j \mid \bar{p}<j^{\prime}\right\}$ which is a contradiction. Hence $m_{1}, \ldots, m_{r}$ are minimal generators of $M$. Thus, $M$ has $r$ generators and it is an ideal in $k\left[x_{1}, \ldots, x_{n}\right]$, where $r$ is the number of atoms of $L$ and $n$ is the number of matroid hyperplanes.

Lemma 2.1. In the notation above, the lcm-lattice of $M$ is $L$.

For the proof of 2.7 we recall some definitions: Let $\Delta$ be a simplicial complex on a vertex set $1, \ldots, n$. Its Stanley-Reisner ideal is

$$
I_{\Delta}=\left(\left\{x_{j_{1}} \cdots x_{j_{p}} \mid\left\{j_{1}, \ldots, j_{p}\right\} \notin \Delta\right\}\right) .
$$

The Alexander dual complex $\Delta^{\vee}$ of $\Delta$ is

$$
\Delta^{\vee}=\left\{[n] \backslash\left\{j_{1}, \ldots, j_{p}\right\} \mid\left\{j_{1}, \ldots, j_{p}\right\} \notin \Delta\right\} .
$$

The minimal monomial generators of $I_{\Delta}$ are

$$
\left\{\frac{x_{1} \ldots x_{n}}{x_{j_{1}} \cdots x_{j_{p}}} \mid\left\{j_{1}, \ldots, j_{p}\right\} \text { is a facet in } \Delta^{\vee}\right\} \text {. }
$$

Proof. Let $\Delta$ be the simplicial complex, such that $M$ is its Stanley-Reisner ideal. Let $\sigma_{1}, \ldots, \sigma_{r}$ be the facets of the Alexander dual complex $\Delta^{\vee}$. The minimal monomial generators of $M$ correspond bijectively to the facets of $\Delta^{\vee}$ via the correspondence

$$
\sigma_{i} \in \Delta^{\vee} \longleftrightarrow \frac{x_{1} \cdots x_{n}}{\prod_{i \in \sigma_{i}} x_{i}} \in M
$$

The equality $\prod_{\bar{i} \vee j^{\prime}=\hat{1}} x_{j}=\frac{x_{1} \cdots x_{n}}{\prod_{i \in \sigma_{i}} x_{i}}$ yields that $\sigma_{i}=\left\{j \mid \bar{i}<j^{\prime}\right\}$. By [GPW, Proposition 2.3(b)] we have that the lcm-lattice of $M$ is isomorphic to the lattice $L^{\prime}$ of intersections of the maximal faces of $\Delta^{\vee}$ enlarged by an additional minimal element $\hat{0}$ and an additional maximal element $\hat{1}$ (the intersections are ordered by reverse inclusion). Note that

$$
\sigma_{i_{1}} \cap \cdots \cap \sigma_{i_{s}}=\left\{j \mid \bar{i}_{p}<j^{\prime}, 1 \leqslant p \leqslant s\right\}=\left\{j \mid \bar{i}_{1} \vee \cdots \vee \bar{i}_{s}<j^{\prime}\right\} .
$$


Now we construct a bijection between $L^{\prime}$ and $L$. Let $F \in L^{\prime}$, consider the correspondence

$$
\bigcap_{F \subseteq \sigma_{i}} \sigma_{i}=F \longleftrightarrow \bigwedge_{j \in F} j^{\prime}=\bigvee_{i<\left(\underset{j \in F}{\wedge} j^{\prime}\right)} \bar{i}=\bigvee_{F \subseteq \sigma_{i}} \bar{i}
$$

We conclude that $L^{\prime}=L$.

Example. Let $L$ be the lattice with atoms $\{\overline{1}\},\{\overline{2}\},\{\overline{3}\},\{\overline{4}\},\{\overline{5}\}$ and coatoms (hyperplanes of the matroid) $\{\overline{1} \overline{2} \overline{3}\},\{\overline{1} \overline{4}\},\{\overline{1} \overline{5}\},\{\overline{2} \overline{4}\},\{\overline{2} \overline{5}\},\{\overline{3} \overline{4} \overline{5}\}$; this lattice is taken from $[\mathrm{Bj} 2,7.6 .1]$. Label these coatoms as $\left\{1^{\prime}\right\},\left\{2^{\prime}\right\},\left\{3^{\prime}\right\},\left\{4^{\prime}\right\},\left\{5^{\prime}\right\},\left\{6^{\prime}\right\}$. Then

$$
M=\left(x_{4} x_{5} x_{6}, x_{2} x_{3} x_{6}, x_{2} x_{3} x_{4} x_{5}, x_{1} x_{3} x_{5}, x_{1} x_{2} x_{4}\right) .
$$

For example, the first generator is $x_{4} x_{5} x_{6}$ because $\left\{1^{\prime}\right\},\left\{2^{\prime}\right\},\left\{3^{\prime}\right\}$ are the coatoms bigger than $\{\overline{1}\}$.

The unsigned Whitney numbers of the first kind coincide with the face numbers of the broken circuit complex by [Bj2, Theorem 7.4.6]. By Theorem 2.2, the unsigned Whitney numbers of the first kind coincide with the Betti numbers of $S / M$. Thus, we can translate [Ox, Conjecture 14.2.7] as

Conjecture 2.1. The sequence $\left\{b_{i}\right\}$ of Betti numbers of $S / M$ is log-concave, i.e.

$$
b_{i}^{2} \geqslant b_{i-1} b_{i+1} \quad \text { for } 1 \leqslant i .
$$

Conjecture 2.9 raises the following problems in the spirit of Stanley's Conjecture $4(\mathrm{~b})$ in [St2]:

Problems 2.1. Let $T$ be an arbitrary monomial ideal and $\left\{b_{i}\right\}$ the sequence of Betti numbers of $S / T$. Find sufficient conditions for $\left\{b_{i}\right\}$ to be log-concave. Find sufficient conditions for $\left\{b_{i}\right\}$ to be unimodal.

One of the most interesting numerical invariants of $\mathcal{A}$ is its Poincaré polynomial $\mathrm{P}_{\mathcal{A}}(t)$. Let $\mu$ be the Möbius function of $L$. The Poincaré polynomial is

$$
\mathrm{P}_{\mathcal{A}}(t)=\sum_{l \in L} \mu(l)(-t)^{\mathrm{rank}(l)} .
$$

Theorem 2.2 implies

Theorem 2.3. $P_{\mathcal{A}}(t)$ is equal to the Poincaré series $P_{S / M}(t)=$ $\sum_{i \geqslant 0} \operatorname{dim} \operatorname{Tor}_{i}(S / M, k) t^{i}$ of the minimal free resolution of $S / M$.

Hilbert's Syzygy Theorem [Ei, 19.7] together with our results implies the following bound: Let $n$ be the number of matroid hyperplanes (maximal flats) of $L$. The broken circuit complex is at most $n$-dimensional.

For $m \in L$ we denote by $(\hat{0}: m)_{L_{M}}$ the open interval $\{l \mid l \in L, \hat{0}<l<m\}$. The homology $\tilde{\mathrm{H}}_{*}\left((\hat{0}: m)_{L} ; k\right)$ is the homology of the abstract simplicial complex with

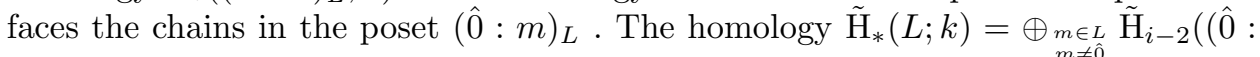
$\left.m)_{L_{M}} ; k\right)$ is called the Whitney homology of the lattice $L$. 
The next corollary was proved in different ways independently by Björner, Gelfand-Zelevinsky, Jambu-Terao. Our proof is new and relies on the exactness of Taylor's resolution (which is easy to prove, see [BPS]), [GPW, Theorem 2.1], and the results in this paper.

Corollary 2.1. Let $b c(L)$ be the broken circuits complex of $L$, and $A$ the OrlikSolomon algebra of $L$. Let $f_{i}$ be the number of $i$-faces in $b c(L)$. Then

$$
f_{i}=\operatorname{dim} A_{i}=\sum_{\substack{m \in L \\ m \neq \hat{0}}} \operatorname{dim} \widetilde{\mathrm{H}}_{i-2}\left((\hat{0}, m)_{L} ; k\right) .
$$

Proof. By construction 2.6 choose a geometric monomial ideal $M$, such that its lcmlattice is $L$. The ideal $M$ and the minimal free resolution of $S / M$ are $\mathbf{N}^{n}$-graded. Therefore we have $\mathbf{N}^{n}$-graded Betti numbers $b_{i, \mathbf{x}^{\alpha}}(S / M)=\operatorname{dim}_{k} \operatorname{Tor}_{i, \alpha}^{S}(S / M, k)$ for $i \geqslant 0, \alpha=\left(\alpha_{1}, \ldots, \alpha_{n}\right) \in \mathbf{N}^{n}$ and $\mathbf{x}^{\alpha}=x_{1}^{\alpha_{1}} \cdots x_{n}^{\alpha_{n}}$. By [GPW, Theorem 2.1], the Whitney homology relates to the Betti numbers of $S / M$ as follows: for $i \geqslant 1$ and $m \in L$ we have

$$
b_{i, m}(S / M)=\operatorname{dim} \widetilde{\mathrm{H}}_{i-2}\left((\hat{0}, m)_{L} ; k\right) .
$$

Hence, for $i \geqslant 1$ we have

$$
b_{i}(S / M)=\sum_{\substack{m \in L \\ m \neq \hat{0}}} \operatorname{dim} \widetilde{\mathrm{H}}_{i-2}\left((\hat{0}, m)_{L} ; k\right) .
$$

Theorem 2.2 implies that $b_{i}(S / M)=f_{i}$. On the other hand, note that $\mathbf{F}_{o s(M)} \otimes$ $k=A$, so Theorem 2.5 implies that $b_{i}(S / M)=\operatorname{dim} A_{i}$.

Below, we introduce a grading on the Orlik-Solomon algebra. Let the atoms of $L$ be labeled by $\overline{1}, \ldots, \bar{r}$ (that is, $\{\overline{1}, \ldots, \bar{r}\}$ is the ground set of the matroid). Let $E$ be the exterior algebra over $\mathbf{C}$ on $n$ generators $e_{1}, \ldots, e_{r}$; this is a differential algebra with differential $d$ acting as $d\left(e_{i_{1}} \wedge \cdots \wedge e_{i_{s}}\right)=\sum_{j=1}^{s}(-1)^{j+1} e_{i_{1}} \wedge \cdots \wedge \hat{e}_{i_{j}} \wedge \cdots \wedge e_{i_{s}}$, (here $\hat{e}_{i_{j}}$ means that this variable is not present in the product). Let $I$ be the ideal in $E$ generated by $\{d$ (circuit) $\}$. Then $A=E / I$ is called the Orlik-Solomon algebra of $L$. If $\mathcal{A}$ is a complex hyperplane arrangement, then the Orlik-Solomon algebra $A$ is isomorphic to $H^{*}\left(\mathbf{C}^{q} \backslash \mathcal{A}, \mathbf{C}\right)$.

Consider the polynomial ring $S=k\left[x_{1}, \ldots, x_{n}\right]$ over the field $k$ as $\mathbf{N}^{n}$-graded by letting $\operatorname{deg}\left(x_{i}\right)$ be the $i^{\text {th }}$ standard basis vector in $\mathbf{N}^{n}$.

Corollary 2.2. If $\mathbf{v}=\left(v_{1}, \ldots, v_{n}\right), \mathbf{u}=\left(u_{1}, \ldots, u_{n}\right) \in \mathbf{N}^{n}$, then we set $\mathbf{v} * \mathbf{u}=$ $\left(\max \left(v_{1}, u_{1}\right), \ldots, \max \left(v_{n}, u_{n}\right)\right)$; this operation makes $\mathbf{N}^{n}$ into a semigroup. The Orlik-Solomon algebra is $\mathbf{N}^{n}$-graded by

$$
\begin{aligned}
\operatorname{deg}\left(e_{i}\right) & =\operatorname{deg}\left(m_{i}\right) \\
\operatorname{deg}\left(e_{i_{1}} \wedge \cdots \wedge e_{i_{s}}\right) & =\operatorname{deg}\left(\operatorname{lcm}\left(m_{i_{1}}, \ldots, m_{i_{s}}\right)\right) .
\end{aligned}
$$

Proof. Clearly, $E$ is $\mathbf{N}^{n}$-graded. Each minimal generator of $I$ has the form $d\left(e_{i_{1}} \wedge\right.$ $\left.\cdots \wedge e_{i_{s}}\right)=\sum_{j=1}^{s}(-1)^{j+1} e_{i_{1}} \wedge \cdots \wedge \hat{e}_{i_{j}} \wedge \cdots \wedge e_{i_{s}}$ with

$$
\bar{i}_{1} \vee \cdots \vee \bar{i}_{s}=\bar{i}_{1} \vee \cdots \vee \bar{i}_{p-1} \vee \bar{i}_{p+1} \vee \cdots \vee \bar{i}_{s} \quad \text { for } 1 \leqslant p \leqslant s
$$


in the intersection lattice $L$. Therefore,

$$
\operatorname{lcm}\left(m_{i_{1}}, \ldots, m_{i_{s}}\right)=\operatorname{lcm}\left(m_{i_{1}}, \ldots, \hat{m}_{p}, \ldots, m_{i_{s}}\right) \text { for } 1 \leqslant p \leqslant s .
$$

This shows that the minimal generators of $I$ are homogeneous. Thus, the quotient $A=E / I$ is $\mathbf{N}^{n}$-graded.

\section{Betti numbers of toric ideals}

Let $\mathcal{A}=\left\{a_{1}, \ldots, a_{n}\right\}$ be a subset of $\mathbf{N}^{d} \backslash\{\mathbf{0}\}, A$ be the matrix with columns $a_{i}$, and suppose that $\operatorname{rank}(A)=d$. Consider the polynomial $\operatorname{ring} S=k\left[x_{1}, \ldots, x_{n}\right]$ over a field $k$ generated by variables $x_{1}, \ldots, x_{n}$ in $\mathbf{N}^{d}$-degrees $a_{1}, \ldots, a_{n}$ respectively. The prime ideal $I_{\mathcal{A}}$, that is the kernel of the homomorphism $k\left[x_{1}, \ldots, x_{n}\right] \rightarrow k\left[t_{1}, \ldots, t_{d}\right]$ mapping $x_{i}$ to $\mathbf{t}^{a_{i}}=t_{1}^{a_{i 1}} \cdots t_{d}^{a_{i d}}$, is called a toric ideal; the ring $S / I_{\mathcal{A}}$ is called a toric ring. Both the polynomial ring $S$ and $I_{\mathcal{A}}$ are $\mathbf{N}^{d}$-graded. If $\alpha \in \mathbf{N}^{d}$, then the set of all monomials in $S$ of degree $\alpha$ is called the fiber of $\alpha$. The minimal free resolution of $S / I_{\mathcal{A}}$ over $S$ is $\mathbf{N}^{d}$-graded as well. We are interested in computing the multigraded Betti numbers

$$
b_{i, \alpha}\left(S / I_{\mathcal{A}}\right)=\operatorname{dim}_{k} \operatorname{Tor}_{i, \alpha}^{S}\left(S / I_{\mathcal{A}}, k\right)
$$

for $i \geqslant 0$ and $\alpha \in \mathbf{N}^{d}$.

In this section $M$ stands for a finite set of monomials $m_{1}, \ldots, m_{r}$ in the polynomial ring $S$. The support $\operatorname{supp}(m)$ of a monomial $m$ is the set $\left\{i \mid x_{i}\right.$ divides $\left.m\right\}$. The $\operatorname{radical} \operatorname{rad}(m)$ is the maximal square-free monomial dividing $m$.

Construction 3.1. Let $X(M)$ be the simplicial complex on vertices the monomials in $M$ and faces $\left\{\left\{m_{j} \mid j \in J\right\} \mid J \subseteq[r], \operatorname{gcd}\left(m_{j} \mid j \in J\right) \neq 1\right\}$.

Construction 3.2. Let $\Gamma(M)$ be the simplicial complex on vertices $x_{1}, \ldots, x_{n}$ and faces the radicals of the monomials in $M$ and all their factors. This complex was introduced in [St].

Furthermore, we introduce the posets $P(M), L_{\mathrm{gcd}}(M)$, and $L_{\sqrt{ }}(M)$, which can be used to compute the Betti numbers:

Let $\bar{P}(M)$ be the lattice with elements the greatest common divisors of monomials in $M$ ordered by reverse divisibility with an additional minimal element $\hat{0}$. The atoms of $\bar{P}(M)$ are $m_{1}, \ldots, m_{r}$. The join operation in $\bar{P}(M)$ is taking gcd. If $1 \in$ $\bar{P}(M)$, then it is the maximal element. We call $\bar{P}(M)$ the gcd-lattice of $M$. Set

$$
L_{\mathrm{gcd}}(M)= \begin{cases}\bar{P}(M) \backslash \hat{0} & \text { if } 1 \notin \bar{P}(M) \\ \bar{P}(M) \backslash\{1, \hat{0}\} & \text { if } 1 \in \bar{P}(M) .\end{cases}
$$

We denote by $\operatorname{Rad}(M)$ the set of the distinct radicals of the monomials in $M$. Set $L_{\sqrt{ }}(M)=L_{\text {gcd }}(\operatorname{Rad}(M))$.

Construction 3.3. We denote by $\operatorname{MaxRad}(M)$ the set consisting of the distinct maximal radicals of monomials in $M$. Set $P(M)=L_{\mathrm{gcd}}(\operatorname{MaxRad}(M))$. We call $P(M) \cup \hat{0}$ the radical lattice of $M$. 
The order complex $\Delta(P)$ of a poset $P$ is the abstract simplicial complex that is the collection of all chains in the poset. Sometimes we implicitly think of $P$ as a topological space by considering its order complex.

The poset $P(M)$ is the smallest and simplest among $L_{\mathrm{gcd}}(M), L_{\sqrt{ }}(M), P(M)$. However, none of the simplicial complexes $X(M), \Gamma(M), \Delta(P(M))$ could be considered as the simplest in general.

Example. Consider the toric surface $R=k\left[u z^{3}, u y^{4} z, u y^{3}, u y^{3} z^{7}, u y^{2} z^{4}\right]$ and the toric ideal that is the kernel of $k[a, b, c, d, e] \rightarrow R$. Take the fiber $M=$ $\left\{a^{5} b^{5} d^{4}, a^{3} d^{5} c^{5} e, a^{2} b d^{3} c^{3} e^{5}, a b^{2} d c e^{9}\right\}$ of $\alpha=u^{14} y^{32} z^{48}$. The poset $P(M)$ consists of the single element abcde. The poset $L_{\sqrt{ }}(M)$ has minimal element abcde, maximal element $a d$, and two rank 1 elements abd and adce. The poset $L_{\mathrm{gcd}}(M)$ is much bigger: it has 11 elements and is not graded. This illustrates that $P(M)$ is much simpler than $L_{\mathrm{gcd}}(M)$ and $L_{\sqrt{ }}(M)$.

For a simplicial complex $\Delta$ on a vertex set $1, \ldots, r$ let $I_{\Delta}$ be the Stanley-Reisner monomial ideal associated to $\Delta$, that is

$$
I_{\Delta}=\left(\left\{x_{j_{1}} \cdots x_{j_{p}} \mid\left\{j_{1}, \ldots, j_{p}\right\} \notin \Delta\right\}\right) .
$$

The Alexander dual complex $\Delta^{\vee}$ of $\Delta$ is

$$
\Delta^{\vee}=\left\{[r] \backslash\left\{j_{1}, \ldots, j_{p}\right\} \mid\left\{j_{1}, \ldots, j_{p}\right\} \notin \Delta\right\} .
$$

Theorem 3.1. - (a) Let $M=m_{1}, \ldots, m_{r}$ be a finite set of monomials. The simplicial complexes $X(M), \Gamma(M)$, and $\Delta(P(M))$ are homotopy equivalent.

- (b) Let $I_{\mathcal{A}}$ be the toric ideal defined by $\mathcal{A}$ and $\alpha \in \mathbf{N}^{d}$. Denote by $M$ the fiber of $\alpha$. Then

$$
\begin{aligned}
b_{i, \alpha}\left(I_{\mathcal{A}}\right) & =\operatorname{dim} \widetilde{\mathrm{H}}_{i}(X(M) ; k) \\
& =\operatorname{dim} \widetilde{\mathrm{H}}_{i}(\Gamma(M) ; k) \\
& =\operatorname{dim} \widetilde{\mathrm{H}}_{i}(P(M) ; k) .
\end{aligned}
$$

- (c) Under the conditions of (c), let $\Delta^{\vee}$ be the Alexander dual complex of $\Gamma(M)$ and $I^{\vee}$ be the Stanley-Reisner ideal of $\Delta^{\vee}$. For $i \geqslant 0$ we have that

$$
b_{i, \alpha}\left(I_{\mathcal{A}}\right)=b_{i, h}\left(I^{\vee}\right),
$$

where $h$ is the square-free product of the variables appearing in the monomials in $M$. The lcm-lattice of $I^{\vee}$ coincides with $P(M)$ enlarged with additional minimal and maximal elements $\hat{0}, \hat{1}$.

Example 3.1. Consider the toric surface $R=k\left[u z^{3}, u y^{4} z, u y^{3}, u y^{3} z^{7}, u y^{2} z^{4}\right]$ and the toric ideal that is the kernel of the map $k[a, b, c, d, e] \rightarrow R$. Take the fiber $M=$ $\left\{a^{4} b^{4} d^{3}, a c^{3} d^{2} e^{5}, b c e^{9}\right\}$ of $\alpha=u^{11} y^{25} z^{37}$. The complex $X(M)$ has the three vertices $a^{4} b^{4} d^{3}, a c^{3} d^{2} e^{5}, b c e^{9}$ and the three edges between them. The complex $\Gamma(M)$ has vertices $a, b, c, d, e$ and facets $b c e, a b d, a c d e$. The poset $P(M)$ has atoms $b c e, a b d, a c d e$ and each of its other elements is equal to the gcd of some couple of atoms. 
Proof of Theorem 3.4. For simplicity, we set $X=X(M), \Gamma=\Gamma(M), L_{\mathrm{gcd}}=$ $L_{\text {gcd }}(M), L_{\sqrt{ }}=L_{\sqrt{ }}(M)$, and $P=P(M)$ throughout the proof. We will show in four steps that $X \simeq L_{\mathrm{gcd}}, L_{\mathrm{gcd}} \simeq L_{\sqrt{ }}, L_{\sqrt{ }} \simeq \Gamma$, and $\Gamma \simeq P$.

The set $M$ is a crosscut in the poset $L_{\mathrm{gcd}}$. The corresponding crosscut complex consists of all bounded subsets of $M$, so it coincides with $X$. By [Bj, Theorem 10.8] the crosscut complex $X$ is homotopy equivalent to the order complex of $L_{\mathrm{gcd}}$.

Consider the map $\varphi: L_{\mathrm{gcd}} \rightarrow L_{\sqrt{ }}$ that sends a monomial to its radical. This map is order preserving. If $p \in L_{\sqrt{ }}$, then the fiber $\varphi^{-1}(p)$ has maximal element $\operatorname{gcd}\left(m_{j} \mid p\right.$ divides $\left.m_{j}, 1 \leqslant j \leqslant r\right)$, hence $\varphi^{-1}(p)$ is contractible. Quillen's fiber lemma [Bj, Theorem 10.5] implies that the order complexes of the posets $L_{\mathrm{gcd}}$ and $L_{\sqrt{ }}$ are homotopy equivalent.

For a monomial $m \in \operatorname{Rad}(M)$ denote by $\Gamma_{m}$ the face $\left\{x_{j} \mid x_{j}\right.$ divides $\left.m\right\}$ of $\Gamma$. Then $\left\{\Gamma_{m} \mid m \in \operatorname{Rad}(M)\right\}$ is a cover of the simplicial complex $\Gamma$. Moreover, if $J \subseteq[r]$ and $\Gamma_{J}=\bigcap_{j \in J} \Gamma_{m_{j}}$ is not empty, then $\Gamma_{J}$ is a full simplex on the vertices $\left\{x_{i} \mid x_{i}\right.$ divides $m_{j}$ for $\left.j \in J\right\}$, so it is contractible. By the nerve lemma $[\mathrm{Bj}$, Theorem 10.6] we get that $\Gamma$ is homotopy equivalent to the nerve simplicial complex $\mathcal{N}$ on vertex set the monomials in $\operatorname{Rad}(M)$ and with faces $\left\{J \subset[r] \mid \Gamma_{J} \neq \emptyset\right\}$. Now note that $\mathcal{N}$ has faces $\left\{J \subset[r] \mid \operatorname{gcd}\left(m_{j} \mid j \in J\right) \neq 1\right\}$. Denote by $L$ the lattice of faces of $\mathcal{N}$ ordered by inclusion with the minimal element removed. Then the order complex $\Delta(L)$ of $L$ is the barycentric subdivision of $\mathcal{N}$. Hence $\Delta(L)$ is homotopic to $\Gamma$. We will show that $L$ and $L_{\sqrt{ }}$ are homotopic. Consider the map $\psi: L \rightarrow L_{\sqrt{ }}$ that sends an element $F$ of $L$ to the monomial $\operatorname{rad}\left(\operatorname{gcd}\left(m_{i} \mid i \in F\right)\right)$. This map is order preserving. If $p \in L_{\sqrt{ }}$, then the fiber $\psi^{-1}(p)$ has maximal element $\bigcap_{i \in H} \Gamma_{m_{i}}$ where $H=\left\{i \mid p\right.$ divides $\left.m_{i}\right\}$, hence $\psi^{-1}(p)$ is contractible. By Quillen's fiber lemma [Bj, Theorem 10.5] it follows that the order complexes of $L$ and $L_{\sqrt{ }}$ are homotopic. Thus, $\Gamma$ is homotopy equivalent to the order complex of $L_{\sqrt{ }}$.

For a monomial $m \in \operatorname{MaxRad}(M)$ denote by $\Gamma_{m}^{\prime}$ the face $\left\{x_{j} \mid x_{j}\right.$ divides $\left.m\right\}$ of $\Gamma$. Then $\left\{\Gamma_{m}^{\prime} \mid m \in \operatorname{MaxRad}(M)\right\}$ is a cover of the simplicial complex $\Gamma$. The same argument as in the above paragraph can be applied to the set $\operatorname{MaxRad}(M)$ instead of to the set $\operatorname{Rad}(M)$; using the cover $\left\{\Gamma_{m}^{\prime} \mid m \in \operatorname{Max} \operatorname{Rad}(M)\right\}$ this argument shows that $\Gamma$ is homotopy equivalent to the order complex of $P$.

The proof of (a) is completed. In order to prove (b) it suffices to show that

$$
b_{i, \alpha}\left(I_{\mathcal{A}}\right)=\operatorname{dim} \widetilde{\mathrm{H}}_{i}(\Gamma ; k) .
$$

This is stated in $[\mathrm{St}]$ and proved in $[\mathrm{AH}]$.

Finally, (c) follows from [GPW, Proposition 2.3(b)] because $P$ is the lattice with elements the intersections of the maximal faces of $\Gamma$ with the minimal element $\hat{0}$ removed.

Remark 3.1. Here we discuss the motivation for Theorem 3.4; it comes from a similar theorem on monomial ideals. Let I be a monomial ideal minimally generated by monomials $m_{1}, \ldots, m_{d}$. The ideal $I$ and the minimal free resolution of $S / I$ over $S$ are $\mathbf{N}^{n}$-graded. Therefore we have $\mathbf{N}^{n}$-graded Betti numbers

$$
b_{i, \mathbf{x}^{\alpha}}(S / I)=\operatorname{dim}_{k} \operatorname{Tor}_{i, \alpha}^{S}(S / I, k)
$$


for $i \geqslant 0, \alpha=\left(\alpha_{1}, \ldots, \alpha_{n}\right) \in \mathbf{N}^{n}$ and $\mathbf{x}^{\alpha}=x_{1}^{\alpha_{1}} \cdots x_{n}^{\alpha_{n}}$. These Betti numbers can be computed using various simplicial complexes: Let $\Gamma(m)$ be the simplicial complex with faces $\left\{J \subseteq[n] \mid \frac{m}{\prod_{i \in J} x_{i}} \in I\right\}$. Denote by $X_{\preceq m}$ the full simplex with vertices the minimal monomial generators of I which divide $m$; let $X(m)$ be the subcomplex of $X_{\preceq m}$ obtained by deleting each face whose vertices have least common multiple equal to $m$. We denote by $L_{I}$ the lattice with elements labeled by the least common multiples of $m_{1}, \ldots, m_{d}$ ordered by divisibility; this is the lcm-lattice of I introduced in $[G P W]$. Set $P(m)$ to be the open interval $(\hat{0}, m)$ in the lattice $L_{I}$.

The next theorem is the motivation for Theorem 3.4.

Theorem 3.2. Let $I$ be a monomial ideal and $m \in L_{I}$.

(a) The simplicial complexes $X(m), \Gamma(m)$, and $\Delta(P(m))$ are homotopy equivalent.

(b) We have

$$
\begin{aligned}
b_{i, m}(I) & =\operatorname{dim} \widetilde{\mathrm{H}}_{i-1}(X(m) ; k) \\
& =\operatorname{dim} \widetilde{\mathrm{H}}_{i-1}(\Gamma(m) ; k) \\
& =\operatorname{dim} \widetilde{\mathrm{H}}_{i-1}(P(m) ; k) .
\end{aligned}
$$

Proof. The proof of [GPW, Theorem 2.1] shows that $X(m)$ and $\Delta(P(m))$ are homotopic; the proof of [BS, Corollary 1.13] shows that $X(m)$ and $\Gamma(m)$ are homotopic; so (a) holds. The first equality for the Betti number in (b) is proved in [BS, Theorem 1.11], the second equality is proved in [BH, Proposition 1.1], and the third is proved in [GPW, Theorem 2.1].

\section{References}

[1] A. Aramova and J. Herzog, Koszul cycles and Eliahou-Kervaire type resolutions, J. Algebra, 181 (1996), 347-370.

[2] D. Bayer, I. Peeva, and B. Sturmfels, Monomial resolutions, Math. Res. Lett. 5 (1998), 31-46.

[3] D. Bayer and B. Sturmfels, Cellular resolutions, J. Reine Angew. Math., 502 (1998), 123-140.

[4] A. Björner, Topological methods, in Handbook of Combinatorics (R. Graham, M. Grötschel, L. Lovász eds.), North-Holland, Amsterdam 1994, 1819 1872.

[5] A. Björner, The homology and shellability of matroids and geometric lattices, in Matroid Applications, Encyclopedia Math. Appl. Cambridge Univ. Press, Cambridge, 40(1992), 226-283.

[6] W. Bruns and J. Herzog, Semigroup rings and simplicial complexes, J. Pure. Appl. Algebra, 122 (1997), 185-208. 
[7] D. Eisenbud, Commutative Algebra with a View Towards Algebraic Geometry, Springer Verlag, New York, 1995.

[8] V. Gasharov, I. Peeva, and V. Welker, The lcm lattice in monomial resolutions, Math. Res. Lett., 6 (1999), 521-532.

[9] G. Lyubeznik, A new explicit finite free resolution of ideals generated by monomials in an R-sequence, J. Pure. Appl. Algebra, 51 (1988), 193-195.

[10] J. Oxley, Matroid theory, Oxford University Press, New York, 1992.

[11] R. Stanley, Combinatorics and Commutative Algebra, Birkhäuser, Boston, 1983.

[12] R. Stanley, Log-concave and unimodal sequences in Algebra, Combinatorics, and Geometry, Graph theory and its applications: East and West, (Jinan, 1986), 500-535, Ann. New York Acad. Sci., 576, New York Acad. Sci., New York, 1989.

This article may be accessed via WWW at http://www.rmi.acnet.ge/hha/ or by anonymous ftp at

ftp://ftp.rmi.acnet.ge/pub/hha/volumes/2002/n2a19/v4n2a19.(dvi,ps,pdf)

Irena Peeva irena@math.cornell.edu

Department of Mathematics,

Purdue University,

West Lafayette, IN 47907 ,

USA 